\title{
LEP Sensitivities to Spontaneous R-Parity Violating Signals
}

\author{
J. C. Romão \\ Inst. Superior Tecnico, Dept. de Física \\ Av. Rovisco Pais, 1 - 1096 Lisboa, Codex, PORTUGAL \\ F. de Campos 0 \\ Instituto de Física Teórica, \\ Universidade Est. Paulista, \\ Rua Pamplona, 145, 01405-900, São Paulo, BRAZIL

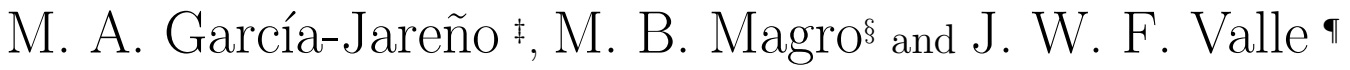 \\ Instituto de Física Corpuscular - C.S.I.C. \\ Departament de Física Teòrica, Universitat de València \\ 46100 Burjassot, València, SPAIN \\ URL: http://neutrinos.uv.es
}

\begin{abstract}
We illustrate the sensitivities of LEP experiments to leptonic signals associated to models where supersymmetry (SUSY) is realized with spontaneous breaking of R-parity. We focus on missing transverse momentum plus acoplanar muon events $\left(\not_{T}+\mu^{+} \mu^{-}\right)$arising from lightest neutralino single production $\chi \nu$ as well as pair production $\chi \chi$, followed by $\chi$ decays, where $\chi$ denotes the lightest neutralino. We show that the integrated luminosity achieved at LEP already starts probing the basic parameters of the theory. We discuss the significance of these constraints for the simplest spontaneous R-parity breaking models and their relevance for future searches of SUSY particles.
\end{abstract}

${ }^{*}$ E-mail fromao@alfa.ist.utl.pt

${ }^{\dagger}$ E-mail fernando@axp.ift.unesp.br

${ }^{\ddagger}$ E-mail miguel@flamenco.ific.uv.es

$\S$ E-mail magro@flamenco.ific.uv.es

`E-mail valle@flamenco.ific.uv.es 


\section{Introduction}

So far most searches for supersymmetric particles have been made in the framework of the Minimal Supersymmetric Standard Model (MSSM) which assumes the conservation of a discrete symmetry called R-parity [目]. Under this symmetry all the standard model particles are R-even while their superpartners are R-odd. R-parity is related to the spin $(\mathrm{S})$, total lepton number $(\mathrm{L})$, and baryon number $(\mathrm{B})$ according to $R_{p}=(-1)^{(3 B+L+2 S)}$. In the limit of exact R-parity the supersymmetric (SUSY) particles must be produced only in pairs, the lightest of them being stable.

Unfortunately there is no clear dynamical clue as to how supersymmetry is realized. In fact, neither gauge invariance nor supersymmetry require the conservation of R-parity. The violation of R-parity could emerge as the residual effect of a more fundamental unified theory [2]. While R-parity violation may be explicit [3], we find it rather attractive to consider the possibility that it arise in a spontaneous way, like the breaking of the electroweak symmetry [⿴囗⿴囗十一]. At the present state-of-the-art theory can not decide. It is therefore of great interest to pursue the phenomenological implications of alternative scenarios. This is specially so in view of the fact that the associated phenomena can be accessible to experimental verification.

Recently there was a lot of attention devoted to the possibility that R-parity can be an exact symmetry of the Lagrangian, broken spontaneously through nonzero vacuum expectation values (VEVS) for scalar neutrinos [5, 6, 0, 8, 9, 10, 11, 12]. There are two main types of scenario [13]. If lepton number is part of the gauge symmetry there is an additional gauge boson which acquires mass via the Higgs mechanism. In this case there is no physical Goldstone boson and the scale of R-parity violation also characterises the new gauge interaction, around the TeV scale [8]. Consequently, its

effects can be large [9]. In this model typically the lightest SUSY particle (LSP) is a neutralino which decays mostly to visible states, breaking R-parity. The main decay modes are three-body,

$$
\chi \rightarrow f \bar{f} \nu
$$

where $\mathrm{f}$ denotes a charged fermion. Its invisible decay modes are in the channel

$$
\chi \rightarrow 3 \nu
$$

Alternatively, if spontaneous R-parity violation occurs in the absence of any additional gauge symmetry, it leads to the existence of a physical massless Nambu-Goldstone boson, called Majoron (J) 14]. Thus in this case the lightest SUSY particle is the Ma- 
joron which is massless and therefore stable . As a result often the lightest neutralino $\chi$ may decay invisibly, conserving R-parity, as

$$
\chi \rightarrow \nu+J .
$$

With the minimal particle content of the MSSM, including only the usual isodoublet sneutrinos, these models lead to a new decay mode for the $Z$ boson, $Z \rightarrow \rho+J$, where $\rho$ is a light scalar with mass $\ll M_{W}$. This decay increases the invisible width of the $Z$ by the equivalent of one half extra neutrino species, and is therefore ruled out by the LEP measurements. This difficulty, as well as the fine-tuning problem characteristic of this simplest scenario, is naturally avoided by adding $S U(2) \otimes U(1)$ singlet neutrinos, in such a way so that the R-parity breaking is driven by the corresponding sneutrino VEVS. In this case the Majoron is mainly singlet [5, 6].

In this paper we analyse some aspects of the phenomenology of spontaneously broken R-parity models at the Z peak. We consider the production of the lightest supersymmetric fermion, including both single as well as pair production mechanisms. In order to identify the corresponding signals we take into account all possible neutralino decay channels, thus extending previous discussions. We focus on the study of events with missing transverse momentum plus acoplanar muons $\left(p_{T}+\mu^{+} \mu^{-}\right)$, arising both from neutralino single production at the $\mathrm{Z}$ peak

$$
Z \rightarrow \chi \nu,
$$

as well as pair production mechanisms

$$
Z \rightarrow \chi \chi
$$

followed by the R-parity violating $\chi$ decays in eq. (18) or eq. (2) and eq. (3). Using the integrated luminosities attained by the four LEP experiments we conclude that the basic parameters of the model are starting to be probed in a meaningful way. We discuss the theoretical significance of these constraints from the point of view of other R-parity-violating processes as well as their possible implications for future SUSY particle searches.

\footnotetext{
"The majoron may have a small mass due to explicit breaking effects at the Planck scale. In this case it may decay to neutrinos and photons. However the time scales are only of cosmological interest and do not change the signal expected at the laboratory [15].
} 


\section{Basic Structure}

Most of our subsequent analysis will be very general and applies to a wide class of $S U(2) \otimes U(1)$ models with spontaneously broken R-parity, such as those of ref. [5, 6], as well as models where the majoron is absent due to an enlarged gauge structure [8, 9. Many of the phenomenological features relevant for the LEP studies discussed here already emerge in an effective model where the violation of R-parity is introduced explicitly through a bilinear superpotential term of the type $\ell H_{u}$ [16.

For concreteness, we start by adopting the conceptually simplest model for the spontaneous violation of R-parity proposed in ref. [5] and by recalling its basic ingredients. This will serve to set up our notation in what follows. We consider this model as the most useful way to parametrise the resulting physics, due to the strict correlation exhibited in this model between the magnitude of R-parity violating phenomena and the $\nu_{\tau}$ mass. For example, all single SUSY particle production rates as well as the lightest neutralino decay rate $\Gamma_{\chi}$ are directly correlated to the mass of the tau neutrino. The superpotential is given by

$$
h_{u} Q H_{u} u^{c}+h_{d} H_{d} Q d^{c}+h_{e} \ell H_{d} e^{c}+\left(h_{0} H_{u} H_{d}-\epsilon^{2}\right) \Phi+h_{\nu} \ell H_{u} \nu^{c}+h \Phi S \nu^{c}+\text { h.c. }
$$

This superpotential conserves total lepton number and R-parity. The superfields ( $\Phi$, $\left.\nu^{c}{ }_{i}, S_{i}\right)$ are singlets under $S U(2) \otimes U(1)$ and carry a conserved lepton number assigned as $(0,-1,1)$ respectively. The couplings $h_{u}, h_{d}, h_{e}, h_{\nu}, h$ are arbitrary matrices in generation space. The additional singlets $\nu^{c}, S$ [17] and $\Phi$ [18] may drive the spontaneous violation of R-parity. This leads to the existence of a Majoron given by the imaginary part of [5]

$$
\frac{v_{L}^{2}}{V v^{2}}\left(v_{u} H_{u}-v_{d} H_{d}\right)+\frac{v_{L}}{V}{\tilde{\nu_{\tau}}}-\frac{v_{R}}{V}{\tilde{\nu^{c}}}_{\tau}+\frac{v_{S}}{V} \tilde{S}_{\tau}
$$

where the isosinglet VEVS

$$
v_{R}=\left\langle\tilde{\nu}_{R \tau}\right\rangle, \quad v_{S}=\left\langle\tilde{S}_{\tau}\right\rangle
$$

with $V=\sqrt{v_{R}^{2}+v_{S}^{2}}$ characterise R-parity or lepton number breaking and the isodoublet VEVS

$$
v_{u}=\left\langle H_{u}\right\rangle, \quad v_{d}=\left\langle H_{d}\right\rangle
$$

are responsible for the breaking of the electroweak symmetry and the generation of fermion masses. The combination $v^{2}=v_{u}^{2}+v_{d}^{2}$ is fixed by the $W, Z$ masses. Finally, there is a small seed of R-parity breaking in the doublet sector, i.e.,

$$
v_{L}=\left\langle\tilde{\nu}_{L \tau}\right\rangle
$$


whose magnitude is now related to the Yukawa coupling $h_{\nu}$. Since this vanishes as $h_{\nu} \rightarrow 0$, we can naturally obey the limits from stellar energy loss [19].

For our subsequent discussion we need the chargino and neutralino mass matrices. The form of the chargino mass matrix is common to a wide class of $S U(2) \otimes U(1)$ SUSY models with spontaneously broken R-parity and is given by

\begin{tabular}{c|ccc} 
& $e_{j}^{+}$ & $\tilde{H}_{u}^{+}$ & $-i \tilde{W}^{+}$ \\
\hline$e_{i}$ & $h_{e i j} v_{d}$ & $-h_{\nu i j} v_{R j}$ & $\sqrt{2} g_{2} v_{L i}$ \\
$\tilde{H}_{d}^{-}$ & $-h_{e i j} v_{L i}$ & $\mu$ & $\sqrt{2} g_{2} v_{d}$ \\
$-i \tilde{W}^{-}$ & 0 & $\sqrt{2} g_{2} v_{u}$ & $M_{2}$
\end{tabular}

Two matrices $\mathrm{U}$ and $\mathrm{V}$ are needed to diagonalise the $5 \times 5$ (non-symmetric) chargino mass matrix

$$
\begin{aligned}
& \chi_{i}^{+}=V_{i j} \psi_{j}^{+} \\
& \chi_{i}^{-}=U_{i j} \psi_{j}^{-}
\end{aligned}
$$

where the indices $i$ and $j$ run from 1 to 5 and $\psi_{j}^{+}=\left(e_{1}^{+}, e_{2}^{+}, e_{3}^{+}, \tilde{H}_{u}^{+},-i \tilde{W}^{+}\right)$and $\psi_{j}^{-}=\left(e_{1}^{-}, e_{2}^{-}, e_{3}^{-}, \tilde{H}_{d}^{-},-i \tilde{W}^{-}\right)$.

Under reasonable approximations, we can truncate the neutralino mass matrix so as to obtain an effective $7 \times 7$ matrix of the following form [5]

\begin{tabular}{c|ccccc} 
& $\nu_{i}$ & $\tilde{H}_{u}$ & $\tilde{H}_{d}$ & $-i \tilde{W}_{3}$ & $-i \tilde{B}$ \\
\hline$\nu_{i}$ & 0 & $h_{\nu i j} v_{R j}$ & 0 & $g_{2} v_{L i}$ & $-g_{1} v_{L i}$ \\
$\tilde{H}_{u}$ & $h_{\nu i j} v_{R j}$ & 0 & $-\mu$ & $-g_{2} v_{u}$ & $g_{1} v_{u}$ \\
$\tilde{H}_{d}$ & 0 & $-\mu$ & 0 & $g_{2} v_{d}$ & $-g_{1} v_{d}$ \\
$-i \tilde{W}_{3}$ & $g_{2} v_{L i}$ & $-g_{2} v_{u}$ & $g_{2} v_{d}$ & $M_{2}$ & 0 \\
$-i \tilde{B}$ & $-g_{1} v_{L i}$ & $g_{1} v_{u}$ & $-g_{1} v_{d}$ & 0 & $M_{1}$
\end{tabular}

This matrix is diagonalised by a $7 \times 7$ unitary matrix $\mathrm{N}$,

$$
\chi_{i}^{0}=N_{i j} \psi_{j}^{0}
$$

where $\psi_{j}^{0}=\left(\nu_{i}, \tilde{H}_{u}, \tilde{H}_{d},-i \tilde{W}_{3},-i \tilde{B}\right)$, with $\nu_{i}$ denoting the three weak-eigenstate neutrinos.

In the above two equations $M_{1,2}$ denote the supersymmetry breaking gaugino mass parameters and $g_{1,2}$ are the $S U(2) \otimes U(1)$ gauge couplings divided by $\sqrt{2}$. We assume the canonical relation $M_{1} / M_{2}=\frac{5}{3} \tan ^{2} \theta_{W}$. Note that the effective Higgsino mixing parameter $\mu$ may be given in some models as $\mu=h_{0}\langle\Phi\rangle$, where $\langle\Phi\rangle$ is the VEV of an appropriate singlet scalar. 
Typical values for the SUSY parameters $\mu, M_{2}$, and the parameters $h_{\nu i, 3}$ lie in the range given by

$$
\begin{array}{cc}
-250 \leq \frac{\mu}{\mathrm{GeV}} \leq 250 & 30 \leq \frac{M_{2}}{\mathrm{GeV}} \leq 1000 \\
10^{-10} \leq h_{\nu 13}, h_{\nu 23} \leq 10^{-1} & 10^{-5} \leq h_{\nu 33} \leq 10^{-1}
\end{array}
$$

while the expectation values lie in the range:

$$
\begin{array}{cc}
v_{L}=v_{L 3}=100 \mathrm{MeV} & v_{L 1}=v_{L 2}=0 \\
50 \mathrm{GeV} \leq v_{R}=v_{R 3} \leq 1000 \mathrm{GeV} & v_{R 1}=v_{R 2}=0 \\
50 \mathrm{GeV} \leq v_{S}=v_{S 3}=v_{R} \leq 1000 \mathrm{GeV} & 1 \leq \tan \beta=\frac{v_{u}}{v_{d}} \leq 30
\end{array}
$$

The diagonalization of eq. (14) gives rise to the mixing of the neutralinos with the neutrinos, leading to R-parity violating gauge couplings. In what follows we will give explicit expressions for the couplings of the SUSY fermions in terms of these diagonalizing matrices.

\section{Charged and Neutral Current Couplings}

Using the above diagonalizing matrices $\mathrm{U}, \mathrm{V}$ and $\mathrm{N}$ one can write the electroweak currents of the mass-eigenstate fermions. For example, the charged current Lagrangian describing the weak interaction between charged lepton/chargino and neutrino/neutralinos may be written as

$$
\frac{g}{\sqrt{2}} W_{\mu} \bar{\chi}_{i}^{-} \gamma^{\mu}\left(K_{L i k} P_{L}+K_{R i k} P_{R}\right) \chi_{k}^{0}+H . C .
$$

where $P_{L, R}$ are the two chiral projectors and the $5 \times 7$ coupling matrices $K_{L, R}$ may be written as

$$
\begin{array}{r}
K_{L i k}=\eta_{i}\left(-\sqrt{2} U_{i 5} N_{k 6}-U_{i 4} N_{k 5}-\sum_{m=1}^{3} U_{i m} N_{k m}\right) \\
K_{R i k}=\epsilon_{k}\left(-\sqrt{2} V_{i 5} N_{k 6}+V_{i 4} N_{k 4}\right)
\end{array}
$$

The matrix $K_{L i k}$ is the analogous of the matrix $K$ introduced in ref [20] and there is a corresponding matrix characterizing right-handed charged currents $K_{R i k}$. These "off-diagonal" blocks corresponding to $i=1 . .3$ and $k=4 . .7$ as well as $i=4,5$ and $k=1 . .3$ are R-parity-breaking couplings.

The corresponding neutral current Lagrangian may be written as

$$
\frac{g}{\cos \theta_{W}} Z_{\mu}\left\{\bar{\chi}_{i}^{-} \gamma^{\mu}\left(O_{L i k}^{\prime} P_{L}+O_{R i k}^{\prime} P_{R}\right) \chi_{k}^{-}+\frac{1}{2} \bar{\chi}_{i}^{0} \gamma^{\mu}\left(O_{L i k}^{\prime \prime} P_{L}+O_{R i k}^{\prime \prime} P_{R}\right) \chi_{k}^{0}\right\}
$$


where the $7 \times 7$ coupling matrices $O_{L, R}^{\prime}$ and $O_{L, R}^{\prime \prime}$ are given by

$$
\begin{array}{r}
O_{L i k}^{\prime}=\eta_{i} \eta_{k}\left(\frac{1}{2} U_{i 4} U_{k 4}+U_{i 5} U_{k 5}+\frac{1}{2} \sum_{m=1}^{3} U_{i m} U_{k m}-\delta_{i k} \sin ^{2} \theta_{W}\right) \\
O_{R i k}^{\prime}=\frac{1}{2} V_{i 4} V_{k 4}+V_{i 5} V_{k 5}-\delta_{i k} \sin ^{2} \theta_{W} \\
O_{L i k}^{\prime \prime}=\frac{1}{2} \epsilon_{i} \epsilon_{k}\left(N_{i 4} N_{k 4}-N_{i 5} N_{k 5}-\sum_{m=1}^{3} N_{i m} N_{k m}\right)=-\epsilon_{i} \epsilon_{k} O_{R i k}^{\prime \prime}
\end{array}
$$

The off-diagonal part of these coupling matrices break R-parity, i.e. when $i=4 . .7$ and $k=1 . .3$ or vice-versa.

In writing these couplings we have assumed CP conservation. Under this assumption the diagonalizing matrices can be chosen to be real. The $\eta_{i}$ and $\epsilon_{k}$ factors are sign factors, related with the relative $\mathrm{CP}$ parities of these fermions, that follow from the diagonalization of their mass matrices.

Like all supersymmetric extensions of the standard model, the spontaneously broken R-parity models are constrained by data that follow from the negative searches for supersymmetric particles at LEP, in particular the most recent limits on chargino masses from the recent run at $130 \mathrm{GeV}$ mass region [21] as well as $\bar{p} p$ collider data gluino production [22].

There are additional restrictions, which are more characteristic of broken R-parity models. They follow from laboratory experiments related to neutrino physics and weak interactions, cosmology and astrophysics [14, 13]. These restrictions play a very important role, as they exclude many parameter choices that are otherwise allowed by the collider constraints, while the converse is not true. The most relevant constraints come from neutrinoless double beta decay and neutrino oscillation searches, direct searches for anomalous peaks at $\pi$ and $\mathrm{K}$ meson decays, the limit on the tau neutrino mass [23], cosmological limits on the $\nu_{\tau}$ lifetime and mass, as well as limits on muon and tau lifetimes, on lepton flavour violating decays and universality violation.

One can perform a sampling of the points in our model which are allowed by all of the above constraints in order to evaluate systematically the attainable value of the couplings [24]. The allowed values for the diagonal (R-parity conserving) couplings for the lightest neutralino $\chi$ and the lightest chargino $\chi^{ \pm}$are of the same order as those in the MSSM.

For the neutral current couplings of the lightest neutralino $\chi$ we can only get an 
upper limit After imposing the experimental constraints explained before, we get

$$
\left|O_{L 44}^{\prime \prime}\right| \lesssim 0.1
$$

In what concerns the R-parity breaking couplings, the biggest ones correspond to the standard lepton belonging to the third family, i.e. $O_{L 43}^{\prime \prime}$, and this will be responsible for the $Z \rightarrow \chi \nu_{\tau}$ decay. This coupling amplitude can reach a few per cent or so for neutralino mass values accessible at LEP [13] and, as we will show, may lead decay rates observable at LEP.

In what follows we will focus on some of the zen-event signals that could be associated to neutralino single as well as pair production and subsequent decays at LEP.

\section{Neutralino Production at the Z Peak}

At the LEP I collider the neutralinos may be produced as

$$
e^{+} e^{-} \rightarrow \chi_{i} \chi_{j}
$$

The differential cross section for these processes including only the $Z$ exchange at $\sqrt{s}=M_{Z}$ is given by

$$
\begin{aligned}
\frac{d \sigma}{d \Omega}\left(e^{+} e^{-} \rightarrow \chi_{i} \chi_{j}\right)= & \frac{\alpha^{2}}{4 s} \frac{1}{2}\left(2-\delta_{i j}\right)|Q(s)|^{2} \lambda^{1 / 2}\left(1, \frac{m_{i}^{2}}{s}, \frac{m_{j}^{2}}{s}\right)\left(\frac{1}{\sin \theta_{w} \cos \theta_{w}}\right)^{4} \\
& {\left[G_{1 i j}(s)+G_{2 i j}(s) \cos \theta+G_{3 i j}(s) \cos ^{2} \theta\right] }
\end{aligned}
$$

where $\lambda$ is the usual Källen function and

$$
\begin{aligned}
& G_{1 i j}(s)=\left(g_{V}^{2}+g_{A}^{2}\right)\left[2 \frac{E_{i}}{\sqrt{s}} \frac{E_{j}}{\sqrt{s}}\left(O_{L i j}^{\prime 2}+O_{R i j}^{\prime \prime 2}\right)+4 \frac{m_{i} m_{j}}{s} O_{L i j}^{\prime \prime} O_{R i j}^{\prime \prime}\right] \\
& G_{2 i j}(s)=2 g_{V} g_{A}\left(O_{L i j}^{\prime \prime 2}-O_{R i j}^{\prime \prime 2}\right) \lambda^{1 / 2}\left(1, \frac{m_{i}^{2}}{s}, \frac{m_{j}^{2}}{s}\right) \\
& G_{3 i j}(s)=\frac{1}{2}\left(g_{V}^{2}+g_{A}^{2}\right)\left(O_{L i j}^{\prime \prime 2}+O_{R i j}^{\prime \prime 2}\right) \lambda\left(1, \frac{m_{i}^{2}}{s}, \frac{m_{j}^{2}}{s}\right)
\end{aligned}
$$

and

$$
\begin{aligned}
& E_{i}=\frac{s+m_{i}^{2}-m_{j}^{2}}{2 \sqrt{s}} ; E_{j}=\frac{s+m_{j}^{2}-m_{i}^{2}}{2 \sqrt{s}} \\
& Q(s)=\frac{s}{s-M_{Z}^{2}+i M_{Z} \Gamma_{Z}}
\end{aligned}
$$


Here $g_{V}$ and $g_{A}$ are the usual vector and axial couplings for the Standard Model $Z e^{+} e^{-}$ vertex

$$
g_{V}=-\frac{1}{4}+\sin ^{2} \theta_{w} \quad ; \quad g_{A}=-\frac{1}{4}
$$

and the relevant $\chi_{i} \chi_{j}$ coupling amplitudes are determined from eq. (24). Notice that, due to the Majorana nature of the neutralinos, their neutral current couplings obey the relation $O_{R i j}^{\prime \prime}=-\epsilon_{i} \epsilon_{j} O_{L i j}^{\prime \prime}$ and therefore the functions $G_{2 i j}(s)$ vanish identically. In the above equations the indices $i$ and $j$ run from 1 to 7 . At $\sqrt{s}=M_{Z}$ they will be restricted only to those corresponding to neutralinos lighter than the $Z$ boson.

As seen above, in models with spontaneously broken R-parity, the mixing of the standard leptons with the supersymmetric charginos and neutralinos leads to the existence of R-violating couplings in the Lagrangian when written in terms of the mass eigenstates. As a result, SUSY particles can be singly-produced. This means that $\chi_{i}$ and $\chi_{j}$ in eq. (26) can be both supersymmetric particles (standard SUSY pair production) as well as one standard and one supersymmetric (R-parity breaking single production). This is in sharp contrast with explicitly broken R-parity models such as considered in ref. [25], where only the pair production of the lightest neutralino $\chi$ can take place at the $\mathrm{Z}$ peak.

In this paper we are therefore concerned with the signals arising from $Z \rightarrow \chi \nu$ decays (corresponding to $i=j=4$ ) and $Z \rightarrow \chi \chi$ decays (corresponding to $i=4, j=$ 3 or vice-versa), where $\chi$ denotes the lightest neutralino. The heavier ones will be assumed to be too heavy to be produced at the $\mathrm{Z}$ peak.

\section{$5 \quad$ Neutralino Decays}

Once produced in $e^{+} e^{-}$collisions, the neutralino subsequently decays, typically inside the detector. In order to identify the expected signatures at LEP it is necessary to specify its possible decay modes. In the MSSM all supersymmetric particles have cascade decays finishing in the LSP which is normally a neutralino. However, if Rparity is broken there are new decay channels and the supersymmetric particles can decay directly to the standard states breaking R-parity. Also the lightest SUSY particle may not be a neutralino, in fact this is the case in spontaneously broken models [5, 6] **. Alternatively, SUSY particles may decay through R-parity conserving cascade decays

${ }^{* *}$ In this case the LSP may be produced in R-parity-violating decays of normal particles like the muon, the tau lepton 11] or the $\mathrm{Z}$ boson 10. 
that will produce the lightest neutralino $\chi$. This may decay invisibly conserving Rparity as in eq. (3), or via the R-parity breaking three fermion modes in eq. (11). To the extent that the invisible dominates, as could happen, one recovers the lightest neutralino missing momentum signal expected in the MSSM.

For simplicity we are going to study the decays of the lightest neutralino, which one expects would be the earliest-produced supersymmetric particle. Heavier states would have cascade decays that we are not going to consider here.

The lightest neutralino $\chi$ has to decay always to standard states breaking $\mathrm{R}$ parity. If its mass is lower than the mass of the gauge bosons it decays to the three body final states. We will distinguish three cases. First all the particles in the final state are neutral. Then

$$
\chi^{0} \rightarrow \nu_{j} \nu_{k} \nu_{m} \quad \text { with width } \quad \Gamma_{1}^{0}=\Gamma^{3 b}\left(M_{\chi^{0}}, O_{L}^{\prime \prime}, O_{R}^{\prime \prime}\right)
$$

In the second case we consider charged leptons in the final state, that is

$$
\chi^{0} \rightarrow \nu_{j} l_{k}^{-} l_{m}^{+} \quad \text { with width } \quad \Gamma_{2}^{0}=\Gamma^{3 b^{\prime}}\left(M_{\chi^{0}}, O_{L}^{\prime \prime}, O_{R}^{\prime \prime}, O_{L}^{\prime}, O_{R}^{\prime}, K_{L}, K_{R}\right)
$$

In both these processes there is interference among the various diagrams contributing. This is clear for the process in Eq. 35 where there is interference between the neutral and charged current diagrams, but it also true for the process in Eq. 34 due to the Majorana nature of the neutrinos. The explicit expressions for the widths $\Gamma^{3 b}$ and $\Gamma^{3 b \prime}$ are given in the Appendix. Finally there is a third case when the final state contains quarks, that is

$$
\chi^{0} \rightarrow \nu_{j} q \bar{q} \quad \text { with width } \quad \Gamma_{3}^{0}=\Gamma^{3 b^{\prime}}\left(M_{\chi^{0}}, O_{L}^{\prime \prime}, O_{R}^{\prime \prime}, O_{L}^{\prime}, O_{R}^{\prime}, 0,0\right)
$$

that proceeds only via neutral current. In the Appendix it is explained how this width can be obtained as a particular case of $\Gamma^{36 \prime}$.

As mentioned in the introduction, the existence of the Majoron implies that in $S U(2) \otimes U(1)$ spontaneously broken R-parity, the neutralino can always decay invisibly eq. (3) with a decay width

$$
\begin{aligned}
& \Gamma_{J j}^{0}=\frac{1}{32 \pi} M_{\chi^{0}}\left(C_{L 4 j}^{2}+C_{R 4 j}^{2}\right) \\
& C_{L i j}=-\epsilon_{i} \epsilon_{j} C_{R i j}=\sum_{k=1}^{3} \epsilon_{j}\left(N_{i k} N_{j 4}+N_{j k} N_{i 4}\right) h_{\nu k 3} \frac{v_{R}}{\sqrt{2} V}
\end{aligned}
$$

Although our discussion will be more general, we neglect, for definiteness, supersymmetric fermion decays mediated by slepton exchange. In this approximation, neutralinos of mass accessible at LEP have only three-body decay modes mediated by charged and neutral currents, except for the two-body majoron decay eq. (3), characteristic of the simplest spontaneous R-parity breaking models. 


\section{Signals at LEP}

In order to study the experimental signals associated to the first kinematically accessible neutral supersymmetric fermions, we have developed an event generator that simulates the processes expected for the LEP collider at $\sqrt{s}=M_{Z}$. It allows us to estimate the detection efficiencies when suitable selection criteria are imposed in order to avoid the expected standard model backgrounds to the processes of interest. We describe below the main steps we follow in order to generate neutralino production and decays. As far as the production is concerned, our generator simulates the following processes at the $Z$ peak:

- a) $e^{+} e^{-} \rightarrow \chi \nu$

- b) $e^{+} e^{-} \rightarrow \chi \chi$

Process a) clearly violates R-parity, so it is a new mode of neutralino production forbidden in the MSSM, as well as in models of explicitly broken R-parity [3] other than those where this violation is due to a bilinear superpotential term $\ell H_{u}$ 《16]. On the other hand, process b) is allowed both in the MSSM as well as in models such as the one used in ref. [3].

The second step of the generation is the decay of the lightest neutralino, which is the most characteristic feature of the R-parity breaking models. As explained in the introduction, if the lightest neutralino $\chi$ is lighter than the $Z$ boson it will have three body decays via charged or neutral currents, as well as the two body invisible decay into neutrino + majoron. These decays produce new supersymmetric signals and the generator allows their detailed study. In contrast, the two-body neutralino decay into neutrino + majoron has as signal missing transverse momentum, because both final particles escape detection and therefore we do not need to generate this process. Thus it suffices for us to generate the three body neutralino decays:

- $\chi \rightarrow \nu_{\tau} Z^{*} \rightarrow \nu_{\tau} l^{+} l^{-}, \nu_{\tau} \nu \nu, \nu_{\tau} q_{i} \overline{q_{i}}$

- $\chi \rightarrow \tau W^{*} \rightarrow \tau \nu_{i} l_{i}, \tau q_{u} \overline{q_{d}}$

The last step of our simulation is made calling the PYTHIA software [26], using as input the neutralino decay products above mentioned.

The signals associated to the first kinematically accessible neutralino, which arise from its single R-parity violating production as well as its R-parity conserving pair 
production are listed in table 1 . This table shows the final signals for the $\chi \nu_{\tau}$ and $\chi \chi$ production with the subsequent $\chi$ decay. One of the cleanest and most interesting signals that can be studied are the events with missing transverse momentum + acoplanar muons $\left(\not p_{T}+\mu^{+} \mu^{-}\right)$. These can be produced through either process a) or process b) as shown in table 1 .

The main source of background for this signal is the $Z$ decay to $\mu^{+} \mu^{-}$with the radiation of a $\gamma$ which may escape detection. This background has to be eliminated through suitable cuts. For definiteness we have imposed the cuts used by the OPAL experiment for their search for acoplanar dilepton events [27]:

- we select events with two muons with at least for one of the muons obeying $|\cos \theta|$ less than 0.7.

- the energy of each muon has to be greater than a $6 \%$ of the beam energy.

- the missing transverse momentum in the event must exceed $6 \%$ of the beam energy, $p_{T}>3 \mathrm{GeV}$.

- the acoplanarity angle (the angle between the projected momenta of the two muons in the plane orthogonal to the beam direction) must exceed $20^{\circ}$.

The OPAL experiment did not find any acoplanar muon pair event passing these cuts in the data sample analysed in ref. [27.

Using our Monte Carlo generator we did the calculation of the kinematic distributions before and after applying these cuts. This was used to determine the detection efficiencies associated to the $\chi \nu$ and $\chi \chi$. They lie in the range $\approx 25 \%-18 \%$ for neutralino masses in the range $m_{\chi} \approx 25 \mathrm{GeV}-40 \mathrm{GeV}$ for the case of $\chi \chi$ production. For the single production case $(\chi \nu)$ we have found efficiencies in the range $11 \%-5 \%$ for $m_{\chi} \approx 40 \mathrm{GeV}-80 \mathrm{GeV}$.

\section{Analysis and Results}

Given a sample of LEP data collected at the $Z$ peak, the study of the signal described in the previous section allows us to determine the corresponding experimental limits on the values of the relevant $R$ parity-violating couplings versus neutralino mass. In order to illustrate the procedure we will use the last data published by the ALEPH collaboration, 
corresponding to an integrated luminosity $L_{\text {int }}=\frac{\text { number hadronic events }}{\sigma\left(e^{+} e^{-} \rightarrow \text { hadrons }\right)}=82 \mathrm{pb}^{-1}$ [25]. This in turn corresponds to $1.94 \times 10^{6}$ hadronic decays of the Z. Of course this is justified only under the assumption that the experimental cuts of the previous section are enough to eliminate all relevant background, or that the detection efficiency for our signal is not reduced by further cuts that might be needed. Clearly, in order to obtain rigorous limits for a given data sample collected in a given experiment, one would have to check whether these assumptions are true by means of more detailed simulation studies of the corresponding detector features as well as the corresponding background for the given luminosity.

While we await for a more complete statistics to be analysed [28] we find it useful to illustrate the sensitivity to the basic parameters of our R-parity violating models which has already been achieved with the data samples collected. For such illustrative purposes we make use only of the cleanest leptonic signal and use the published ALEPH data given in ref. [25]. As usual, in order to obtain a $95 \% C L$ limit on some parameter, we impose the condition

$$
3>N_{\text {expt }}
$$

$N_{\text {expt }}$ being the number of expected events for our signal, when no events of the desired type have been observed.

For the single production process $e^{+} e^{-} \rightarrow \chi \nu$ with the decay $\chi \rightarrow \nu_{\tau} \mu^{+} \mu^{-}$the expected number $p_{T}+\mu^{+} \mu^{-}$events is given as

$$
N_{\text {expt }}(\chi \nu)=\sigma\left(e^{+} e^{-} \rightarrow \chi \nu\right) B R\left(\chi \rightarrow \nu_{\tau} \mu^{+} \mu^{-}\right) \epsilon_{\chi \nu} L_{i n t}
$$

where $\epsilon_{\chi \nu}$ is the detection efficiency, obtained from the R-parity breaking generator described before.

Using the expression for the cross section in eq. (27) we can write

$$
\begin{aligned}
N_{\text {expt }}(\chi \nu)= & \frac{2}{3} O_{L 43}^{\prime \prime 2} \frac{\alpha^{2} \pi\left(g_{V}^{2}+g_{A}^{2}\right)}{\Gamma_{Z}^{2}\left(\sin \theta_{w} \cos \theta_{w}\right)^{4}}\left(2-3 x_{Z}^{2}+x_{Z}^{6}\right) \\
& B R\left(\chi \rightarrow \nu_{\tau} \mu^{+} \mu^{-}\right) \epsilon_{\chi \nu} L_{\text {int }}
\end{aligned}
$$

where $x_{Z}=m_{\chi} / m_{Z}$.

In addition, the relation between the coupling $O_{L 43}^{\prime \prime 2}$ and the $B R(Z \rightarrow \chi \nu)$ is given by

$$
B R(Z \rightarrow \chi \nu)=\frac{2}{3} O_{L 43}^{\prime \prime 2} \frac{M_{Z}^{3} G_{F}}{\Gamma_{Z} \pi \sqrt{2}}\left(1-\frac{3}{2} x_{Z}^{2}+\frac{1}{2} x_{Z}^{6}\right)
$$


From eq. (40), eq. (41) and eq. (38) one can obtain a 95\%CL limit on the R-parity breaking observable $B R(Z \rightarrow \chi \nu) B R\left(\chi \rightarrow \nu_{\tau} \mu^{+} \mu^{-}\right)$as a function of the $\chi$ mass. This is shown in figures 2 and 4 .

Here we should stress that this production mode is characteristic of models with spontaneous violation of R-parity [5, 6, 9], or models that parametrize it through an effective bilinear superpotential term $\epsilon \ell H$ [16]. It is absent in most models of explicitly broken R-parity, such as the one considered in the analysis presented by the ALEPH collaboration in ref. 25.].

Notice that the R-parity-violating parameter $\epsilon$ is directly correlated to the mass of the tau neutrino $\nu_{\tau}$. As a result it is correspondingly restricted by cosmological Big Bang nucleosynthesis [30]. The corresponding limits are rather stringent [31] and may not allow $m_{\nu_{\tau}}$ above a few hundred $\mathrm{KeV}$. In this case the signal displayed in Fig. 4 would never reach $10^{-8}$. This cosmological bound can be avoided in models which contain neutrino decay [32] or annihilation channels [33] beyond those present within the standard model. For this reason the spontaneously broken R-parity models are preferred, as they allow the maximum $m_{\nu_{\tau}}$ values permitted by laboratory experiments 23. due to the $\nu_{\tau}$ decays and/or annihilations to majorons. However, as seen in Fig. 4 , the maximum value of our signal is only a bit larger than $10^{-7}$.

We conclude that in models such as the ones in ref. [16, 9] one can probe the spontaneous violation of R-parity in the single-production mode for $m_{\chi} \approx 40 \mathrm{GeV}$. However, as already mentioned, these values of the signal rate are hard to reconcile with cosmological $\nu_{\tau}$ limits. The corresponding rates for a cosmologically acceptable $\nu_{\tau}$ mass lie well bellow the dotted curve in Fig. (4), below $10^{-8}$ for all values of the neutralino mass, thus too small a rate to be of interest. Including the majoron, whose existence is expected in any model where the spontaneous violation of R-parity is realized in the minimal $S U(2) \otimes U(1)$ gauge structure [5, 6], changes the situation in two ways. First, it improves the allowed signal rates because it allows $\nu_{\tau}$ masses as large as present laboratory limits. Unfortunately there is a counter-effect that decreases the expected signal rates, because the presence of the invisible channel eq. (3) tends to dilute the $\chi$ branching ratio into muons. The net result leads to the dotted curve in Fig. (4).

The lightest neutralino $\chi$ may however be light enough to be pair-produced in the standard R-parity-conserving process $e^{+} e^{-} \rightarrow \chi \chi$. Even in this case of standard production, the violation of R-parity can provide visible signals from the subsequent 
decays of the neutralinos. The first possibility to consider here is the case where both neutralinos decay visibly, e.g. into, $\nu_{\tau} \mu^{+} \mu^{-}$. We do not consider this possibility as it is similar to the one used recently by ALEPH and the corresponding sensitivities may be estimated by re-scaling the results of ref. [25]. Thus we choose concentrate on the novel possibility that one of the neutralinos decays to $\nu_{\tau} \mu^{+} \mu^{-}$while the other decays invisibly, which is more characteristic of models with spontaneous violation of R-parity. The number of expected $\not p_{T}+\mu^{+} \mu^{-}$events in this case is given by

$$
N_{\text {expt }}(\chi \chi)=\sigma\left(e^{+} e^{-} \rightarrow \chi \chi\right) 2 B R(\chi \rightarrow \text { invisible }) B R\left(\chi \rightarrow \nu_{\tau} \mu^{+} \mu^{-}\right) \epsilon_{\chi \chi} L_{\text {int }}
$$

so that from eq. (27) we obtain

$$
\begin{aligned}
N_{\text {expt }}(\chi \chi)= & \frac{2}{3} O_{L 44}^{\prime \prime 2} \frac{\alpha^{2}}{\Gamma_{Z}^{2}} \frac{\pi\left(g_{V}^{2}+g_{A}^{2}\right)}{\left(\sin \theta_{w} \cos \theta_{w}\right)^{4}}\left(1-4 x_{Z}^{2}\right)^{3 / 2} \\
& 2 B R\left(\chi \rightarrow \nu_{\tau} \mu^{+} \mu-\right) B R(\chi \rightarrow \text { invisible }) \epsilon_{\chi \chi} L_{\text {int }}
\end{aligned}
$$

and the corresponding expression for the $Z \rightarrow \chi \chi$ branching ratio is

$$
B R(Z \rightarrow \chi \chi)=\frac{1}{3} O_{L 44}^{\prime \prime 2} \frac{M_{Z}^{3} G_{F}}{\Gamma_{Z} \pi \sqrt{2}}\left(1-4 x_{Z}^{2}\right)^{3 / 2}
$$

From these last expressions and eq. (38) it is possible to obtain an illustrative $95 \% C L$ limit on $B R(Z \rightarrow \chi \chi) B R\left(\chi \rightarrow \nu_{\tau} \mu^{+} \mu^{-}\right) B R(\chi \rightarrow$ invisible $)$ as a function of the $\chi$ mass, as shown in figures 3 and 5 .

\section{Discussion}

Using the integrated luminosity corresponding to the last published ALEPH data, $L=$

$82 p b^{-1}$, we have illustrated how the existing data gathered by the LEP collaborations at the $\mathrm{Z}$ peak are sufficient to start probing in a theoretically meaningful way the mass and couplings of the lowest lying neutral supersymmetric fermions in spontaneously broken R-parity models. We have determined the corresponding regions of sensitivity, illustrated in fig. 2-5, both for the case of single production as well as for the case where the lightest neutralino can be pair produced. The theoretical significance of these decays is illustrated in Fig. (6).

From curve b in this Fig. (代) one sees that the expected signal rate for a neutralino of $40 \mathrm{GeV}$ in the model of ref. [5] could be tested if the luminosities of the four LEP experiments are added, even if only leptonic $p_{T}+\mu^{+} \mu^{-}$and $p_{T}+e^{+} e^{-}$channels 
are considered. This constraint would be significant from the point of view of the underlying model. Indeed, as can be seen from Fig. (6), this corresponds to values of the $\nu_{\tau}$ mass close to an $\mathrm{MeV}$, for which other processes such as $Z \rightarrow \chi^{ \pm}+\tau^{\mp}$ [7, 12] would be expected to be sizeable. Although the presence of the majoron leads to novel decays of standard model particles, e.g. $\mu \rightarrow e+J$ and $\tau \ell+J$ decays [11, 12] of relevance for intense muon source studies at PSI or for a future tau-charm factory [29], as well as novel $Z$ decays such as $Z \rightarrow \gamma+J$ [10, its overall effect insofar as the signal rate for $p_{T}+\mu^{+} \mu^{-}$events arising from $\chi \nu$ production at the $\mathrm{Z}$ peak is concerned is to decrease. One advantage is that the rate there is no conflict between the signal rate (dotted curve in Fig. 3) and the primordial Helium abundance. However, there is clearly a wide range of parameters still unconstrained by this single production mechanism. In contrast, if kinematically accessible, neutralino pair-production can place more severe restrictions, especially on models where the spontaneous violation of R-parity where the majoron is absent [8] or in an effective model where the violation of R-parity is introduced explicitly via the bilinear superpotential term $\ell H_{u}$ [16]. The constraints derived in this case should be important in relation to searches at higher energies such as LEP200, the NLC or the LHC where one expects mostly SUSY pair production to yield sizeable event rates.

Substantial improvements are expected from the use of $e^{+} e^{-}, \tau^{+} \tau^{-}$and di-jet plus missing momentum event topologies. The results presented here should encourage one to perform more detailed and complete background studies and simulations covering other R-parity violating signals and improved integrated luminosities already attained at LEP [28]. From our point of view it would be desirable to have additional runs at the $\mathrm{Z}$ peak, considering the fact that large areas of parameter space still remain open, where the neutralinos are light enough to be produced in $\mathrm{Z}$ decays.

\section{Acknowledgements}

We thank Mario Pimenta for useful discussions. This work was supported by

DGICYT under grant number PB92-0084 and by Acción Integrada Hispano-Portuguesa HP-53B, as well as by DGICYT (M. A. G. J.) and CNPq (M. B. M. and F. de Campos) Fellowships.

\section{Appendix}




\section{A 3-Body Neutralino Decays}

In models with spontaneous R-parity violation, the neutral fermions eigenstates are mixed. We denote them collectively by $\chi_{i}^{0}$ with $(i=1, \ldots, 7)$. The neutrinos correspond in this notation to $i=1,2,3$ and the usual neutralinos to $i=4, \ldots, 7$. In the

charged fermion sector, the leptons are mixed with the charginos of the MSSM. We denote them by $\chi_{i}^{-}$with $(i=1, \ldots, 5)$. Again, the indices $(i=1,2,3)$ correspond, respectively to $e^{-}, \mu^{-}$and $\tau^{-}$, while the indices $i=4,5$ denote the usual charginos. The quarks do not mix with the other fermions. We will consider separately the cases where all the particles in the final state have the same charge and the case where they can have different charges.

\section{A.1 Same-Charge Final States}

Here the decay we consider is into neutral final states

$$
\chi_{i}^{0} \rightarrow \chi_{j}^{0}+\chi_{k}^{0}+\chi_{m}^{0}
$$

and proceeds via the neutral current. Due to the Majorana nature of the neutral fermions, there are 3 distinct diagrams giving rise to some interference terms. The final result for the decay, within the approximation that we neglect all masses of the final fermions, is given by

$$
\begin{aligned}
\Gamma^{3 b}\left(M_{i}, O_{L}^{\prime \prime}, O_{R}^{\prime \prime}\right)=\frac{G_{F}^{2} M_{i}^{5}}{48 \pi^{3}}[ & \left(c_{1}+c_{2}+c_{3}\right) f\left(x_{Z}\right) \\
& \left.+2\left(c_{12}+c_{13}+c_{23}\right) g\left(x_{Z}, x_{Z}\right)\right] \frac{1}{S_{F}}
\end{aligned}
$$

where

$$
\begin{aligned}
& c_{1}=\left(O_{L j i}^{\prime \prime 2}+O_{R j i}^{\prime \prime 2}\right)\left(O_{L k m}^{\prime \prime 2}+O_{R k m}^{\prime \prime 2}\right) \\
& c_{2}=\left(O_{L k i}^{\prime \prime 2}+O_{R k i}^{\prime \prime 2}\right)\left(O_{L j m}^{\prime \prime 2}+O_{R j m}^{\prime \prime 2}\right) \\
& c_{3}=\left(O_{L k j}^{\prime \prime 2}+O_{R k j}^{\prime \prime 2}\right)\left(O_{L i m}^{\prime \prime 2}+O_{R i m}^{\prime \prime 2}\right) \\
& c_{12}=O_{L k m}^{\prime \prime} O_{L j i}^{\prime \prime} O_{L k i}^{\prime \prime} O_{L j m}^{\prime \prime}+O_{R k m}^{\prime \prime} O_{R j i}^{\prime \prime} O_{R k i}^{\prime \prime} O_{R j m}^{\prime \prime} \\
& c_{13}=-\left(O_{L k m}^{\prime \prime} O_{R j i}^{\prime \prime} O_{L k j}^{\prime \prime} O_{L i m}^{\prime \prime}+O_{R k m}^{\prime \prime} O_{L j i}^{\prime \prime} O_{R k j}^{\prime \prime} O_{R i m}^{\prime \prime}\right) \\
& c_{23}=O_{L k i}^{\prime \prime} O_{L k j}^{\prime \prime} O_{R j m}^{\prime \prime} O_{R i m}^{\prime \prime}+O_{R k i}^{\prime \prime} O_{R k j}^{\prime \prime} O_{L j m}^{\prime \prime} O_{L i m}^{\prime \prime}
\end{aligned}
$$

$S_{F}$ is the symmetry factor for identical particles in the final state, $x_{Z}=M_{i} / M_{Z}$, and the functions $f(x)$ and $g(x, y)$ are given in the Appendix of ref. [24]. The coupling matrices are given in section 3 . 


\section{A.2 Different-Charge Final States}

In this case we consider 3-body final states where two are oppositely charged, that is

$$
\chi_{i}^{0} \rightarrow \chi_{j}^{0}+\chi_{k}^{-}+\chi_{m}^{+}
$$

This decay can proceed via both charged and neutral current. Also in the charged current case, the Majorana nature of the neutralino implies the existence of two distinct diagrams. The final result for the decay, within the approximation that we neglect all masses of the final fermions, is given by

$$
\begin{aligned}
& \Gamma^{3 b^{\prime}}\left(M_{i}, O_{L}^{\prime \prime}, O_{R}^{\prime \prime}, O_{L}^{\prime}, O_{R}^{\prime}, K_{L}, K_{R}\right) \\
& =\frac{G_{F}^{2} M_{i}^{5}}{48 \pi^{3}}\left[\begin{array}{c}
c_{1} f\left(x_{Z}\right)+\left(c_{2}+c_{3}\right) f\left(x_{W}\right) \\
\left.\quad+2\left(c_{12}+c_{13}\right) g\left(x_{Z}, x_{W}\right)+2 c_{23} g\left(x_{W}, x_{W}\right)\right]
\end{array}\right.
\end{aligned}
$$

where $x_{W}=M_{i} / M_{W}$, the functions $f(x)$ and $g(x, y)$ are as before, and the coefficients $c_{i}$ are now given by

$$
\begin{aligned}
c_{1} & =\left(O_{L j i}^{\prime \prime 2}+O_{R j i}^{\prime \prime 2}\right)\left(O_{L k m}^{\prime 2}+O_{R k m}^{\prime 2}\right) \\
c_{2} & =\left(Y_{L k i}^{2}+Y_{R k i}^{2}\right)\left(X_{L j m}^{2}+X_{R j m}^{2}\right) \\
c_{3} & =\left(Y_{L k j}^{2}+Y_{R k j}^{2}\right)\left(X_{L i m}^{2}+X_{R i m}^{2}\right) \\
c_{12} & =O_{L k m}^{\prime} O_{L j i}^{\prime \prime} Y_{L k i} X_{L j m}+O_{R k m}^{\prime} O_{R j i}^{\prime \prime} Y_{R k i} X_{R j m} \\
c_{13} & =-\left(O_{L k m}^{\prime} O_{R j i}^{\prime \prime} Y_{L k j} X_{L i m}+O_{R k m}^{\prime} O_{L j i}^{\prime \prime} Y_{R k j} X_{R i m}\right) \\
c_{23} & =Y_{L k i} Y_{L k j} X_{R j m} X_{R i m}+Y_{R k i} Y_{R k j} X_{L j m} X_{L i m}
\end{aligned}
$$

with

$$
\begin{aligned}
Y_{L i j} & =\frac{1}{\sqrt{2}} K_{L i j} \\
Y_{R i j} & =\frac{1}{\sqrt{2}} K_{R i j} \\
X_{L i j} & =\frac{1}{\sqrt{2}} K_{L j i} \\
X_{R i j} & =\frac{1}{\sqrt{2}} K_{R j i}
\end{aligned}
$$

In all the previous expressions, the coupling matrices are taken to be real. The sign factors $\epsilon_{i}$ and $\eta_{i}$ are introduced as explained in section 3 .

Before we close this discussion, we notice that Eq. 49 can also be applied, with obvious modifications, to the case of quarks in the final state. For definiteness consider the 
process

$$
\chi_{i}^{0} \rightarrow \chi_{j}^{0}+u+\bar{u}
$$

It is clear that in this case we have only the $Z$ exchange diagram. The formula of Eq. 49 it is still valid with the following values for the coefficients $c_{i}$

$$
\begin{aligned}
& c_{1}=\left(O_{L j i}^{\prime \prime 2}+O_{R j i}^{\prime \prime 2}\right)\left[\left(\frac{1}{2}-Q_{u} \sin ^{2} \theta_{w}\right)^{2}+\left(-Q_{u} \sin ^{2} \theta_{w}\right)^{2}\right] \\
& c_{2}=c_{3}=c_{12}=c_{13}=c_{23}=0
\end{aligned}
$$

Notice that the neutralino decay formulas given above correct those previously given in the Appendix of ref. [24, which were not correct for the case of decays involving Majorana fermions, such as $\chi \rightarrow 3 \nu_{\tau}$. However the functions $f(x)$ and $g(x, y)$ are the same as in ref. 224. 


\section{References}

[1] H. P.Nilles, Phys. Rep. 110 (1984) 1; H. Haber and G. Kane, Phys. Rep. 117 (1985) 75

[2] L. Hall, M. Suzuki, Nucl. Phys. B231 (1984) 419

[3] S. Dimopoulos, L.J. Hall, Phys. Lett. 207B (1988) 210; E. Ma, D. Ng, Phys. Rev. D41 (1990) 1005; V. Barger, G. F. Giudice, T. Han, Phys. Rev. D40 (1989) 2987; T. Banks, Y. Grossman, E. Nardi, Y. Nir, Phys. Rev. D52 (1995) 5319; M. Nowakowski, A. Pilaftsis, hep-ph/9508271; B. de Carlos, P. L. White, OUTP-96$01 \mathrm{P}$

[4] C. S. Aulakh, R.N. Mohapatra, Phys. Lett. B119 (1982) 136; G. G. Ross, J. W. F. Valle, Phys. Lett. 151B (1985) 375; J. Ellis, G. Gelmini, C. Jarlskog, G.G. Ross, J.W.F. Valle, Phys. Lett. 150B (1985) 142; A. Santamaria, J.W.F. Valle, Phys. Lett. 195B (1987) 423; Phys. Rev. D39 (1989) 1780; Phys. Rev. Lett. 60 (1988) 397

[5] A Masiero, J. W. F. Valle, Phys. Lett. B251 (1990) 273; J. C. Romão, C. A. Santos, and J. W. F. Valle, Phys. Lett. B288 (1992) 311

[6] G. Giudice, A. Masiero, M. Pietroni, A. Riotto, Nucl. Phys. B396 (1993) 243; M. Shiraishi, I. Umemura, K. Yamamoto, Phys. Lett. B313 (1993) 89; see also I. Umemura, K. Yamamoto, Nucl. Phys. B423 (1994) 405

[7] P. Nogueira, J. C. Romão, J. W. F. Valle, Phys. Lett. B251 (1990) 142 R. Barbieri, L. Hall, Phys. Lett. B238 (1990) 86.

[8] J. W. F. Valle, Phys. Lett. B196 (1987) 157

[9] M. C. Gonzalez-Garcia, J W F Valle, Nucl. Phys. B355 (1991) 330.

[10] J. Romão, J. Rosiek and J. W. F. Valle, Phys. Lett. B351 (1995) 497

[11] J. C. Romão, N. Rius, J. W. F. Valle, Nucl. Phys. B363 (1991) 369.

[12] J. C. Romão and J. W. F. Valle. Phys. Lett. B272 (1991) 436; Nucl. Phys. B381 (1992) 87.

[13] For a recent review see J. W. F. Valle, in Physics Beyond the Standard Model, lectures given at the VIII Jorge Andre Swieca Summer School (Rio de Janeiro, 
February 1995) and at $V$ Taller Latinoamericano de Fenomenologia de las Interacciones Fundamentales (Puebla, Mexico, October 1995); hep-ph/9603307

[14] For recent reviews see J. W. F. Valle, Gauge Theories and the Physics of Neutrino Mass, Prog. Part. Nucl. Phys. 26 (1991) 91-171 (ed. A. Faessler), and G. Gelmini and S. Roulet, UCLA/94/TEP/36 and references therein

[15] V. Berezinskii and J.W.F. Valle, Phys. Lett. B318 (1993) 360; A. D. Dolgov, S. Pastor, and J.W.F. Valle astro-ph/9506011

[16] F. de Campos, M. A. Garcia-Jareño, A. Joshipura, J. Rosiek, J. W. F. Valle, Nucl. Phys. B451 (1995) 3-15; H. Hempfling, MPI-PhT/95-59

[17] R. Mohapatra, J. W. F. Valle, Phys. Rev. D34 (1986) 1642; J. W. F. Valle, Theory and Implications of Neutrino Mass, Nucl. Phys. B (Proc. Suppl.) 11 (1989) 118-177 and references therein.

[18] R. Barbieri, S. Ferrara, and C. Savoy, Phys. Lett. B119, 343 (1982).

[19] J. E. Kim, Phys. Rep. 150 (1987) 1 D. Dearborn, et al, Phys. Rev. Lett. 56 (1986) 26; M. Fukugita et al, Phys. Rev. Lett. 48 (1982) 1522; Phys. Rev. D26 (1982) 1841; J. Ellis, K. Olive, Nucl. Phys. B223 (1983) 252

[20] J. Schechter, J. W. F. Valle, Phys. Rev. D22 (1980) 2227

[21] ALEPH collaboration, CERN-PPE/96-10, submitted to Phys. Lett. B (1996) .

[22] D0 collaboration, Phys. Rev. Lett. 75 (1995) 618; and references therein, CDF collaboration, Phys. Rev. Lett. 69 (1992) 3439; UA2 collaboration, Phys. Lett. B235 (1990) 363.

[23] D. Buskulic et al., Phys. Lett. B349 (1995) 585.

[24] M. C. Gonzalez-Garcia, J. C. Romão, J. W. F. Valle, Nucl. Phys. B391 (1993) 100

[25] D. Buskulic et al Phys. Lett. B349 (1995) 238

[26] T. Sjostrand, Comp. Phys. Commun. 82 (1994) 74

[27] P. D. Acton et al Phys. Lett. B273 (1991) 338

[28] F. de Campos et al, in preparation 
[29] J. W. F. Valle, in Proceedings of Second Workshop on Tau-Lepton Physics, Ohio, ed. K. K. Gan (World Scientific, 1993); see also Third Workshop on the Charm Tau Factory, Marbella, Spain, June 1993, (World Scientific, 1994), Ed. J. Kirkby and R. Kirkby.

[30] N. Hata, et al Phys. Rev. Lett. 75 (1995) 3977; C. J. Copi, D. N. Schramm and M. S. Turner, Science 267 (1995) 192; for a recent review see S. Sarkar, Oxford preprint OUTP-95-16P

[31] E.W. Kolb, M.S. Turner, A. Chakravorty and D.N. Schramm, Phys. Rev. Lett. 67 (1991) 533; A.D. Dolgov and I.Z. Rothstein, Phys. Rev. Lett. 71 (1993) 476. A. Dolgov, S. Pastor, and J. W. F. Valle, hep-ph/9602233

[32] M. Kawasaki, G. Steigman and H.-S. Kang, Nucl. Phys. B402 (1993) 323, Nucl. Phys. B419 (1994) 105; S. Dodelson, G. Gyuk and M.S. Turner, Phys. Rev. D49 (1994) 5068

[33] A.D. Dolgov, S. Pastor, J.C. Romão and J.W.F. Valle, in preparation; S. Pastor and J. W. F. Valle, talks at TAUP95, Toledo, hep-ph/9602369 


\begin{tabular}{||l|l||}
\hline \hline$\chi \chi$ & $\chi \nu$ \\
\hline$p_{T}+l_{i}^{+} l_{i}^{-}+l_{j}+l_{j}-$ & $p_{T}+l_{i}^{+} l_{i}^{-}$ \\
$p_{T}+l_{i}^{+} l_{i}^{-}+2 j e t s$ & $p_{T}+(\tau l)$ \\
$p_{T}+l_{i}^{+} l_{i}^{-}+(2 j e t s+\tau)$ & $p_{T}+\tau+2 j e t s$ \\
$p_{T}+l_{i}^{+} l_{i}^{-}+(\tau l)$ & $p_{T}+2 j e t s$ \\
$p_{T}+l_{i}^{+} l_{i}^{-}$ & \\
$p_{T}+4 j e t s$ & \\
$p_{T}+4 j e t s+\tau$ & \\
$p_{T}+2 j e t s+(\tau l)$ & \\
$p_{T}+2 j e t s$ & \\
$4 j e t s+\tau \tau$ & \\
$p_{T}+2 j e t s+\tau$ & \\
$p_{T}+2 j e t s+\tau(\tau l)$ & \\
$p_{T}(\tau l)(\tau l)$ & \\
$p_{T}(\tau l)$ & \\
\hline \hline
\end{tabular}

Table 1: Final signals arising from neutralino pair-production (left column) as well single production (right column). The total charge of the particles between parenthesis must be zero.

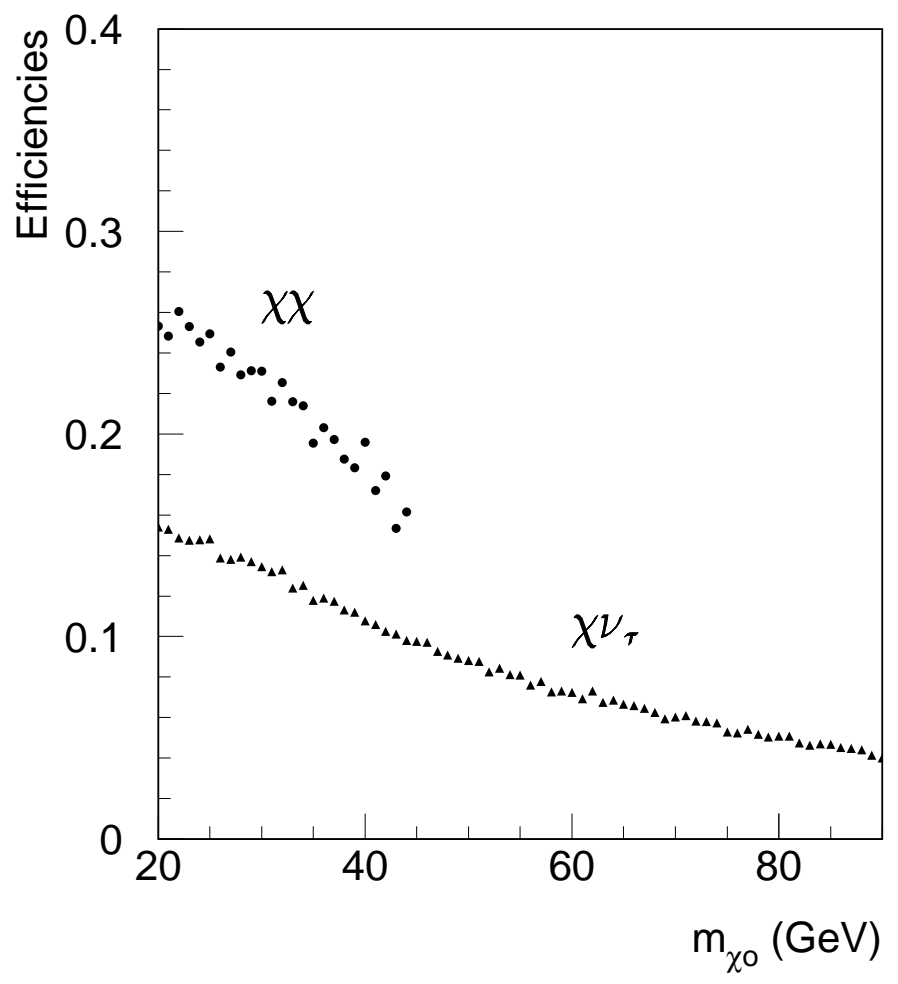

Figure 1: Detection efficiencies for the $\not p_{T}+\mu^{+} \mu^{-}$signal associated to $\chi \nu$ and $\chi \chi$ production channels. 


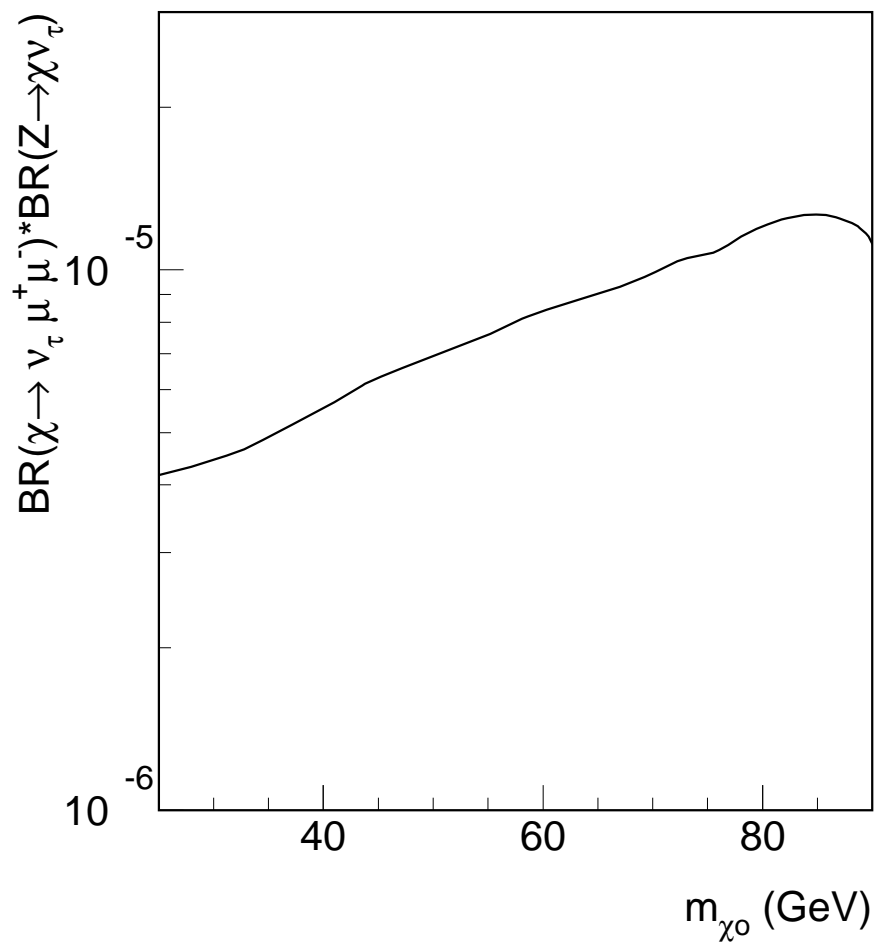

Figure 2: Region of sensitivity obtained at the $95 \%$ C.L. for $B R(Z \rightarrow \chi \nu) B R(\chi \rightarrow$ $\mu^{+} \mu^{-} \nu$ ), as a function of the lightest neutralino mass $m_{\chi^{0}}$. This is derived from searches of $p_{T}+\mu^{+} \mu^{-}$events that would arise from single neutralino production at LEP, followed by $\chi \rightarrow \mu^{+} \mu^{-} \nu$ decays. 


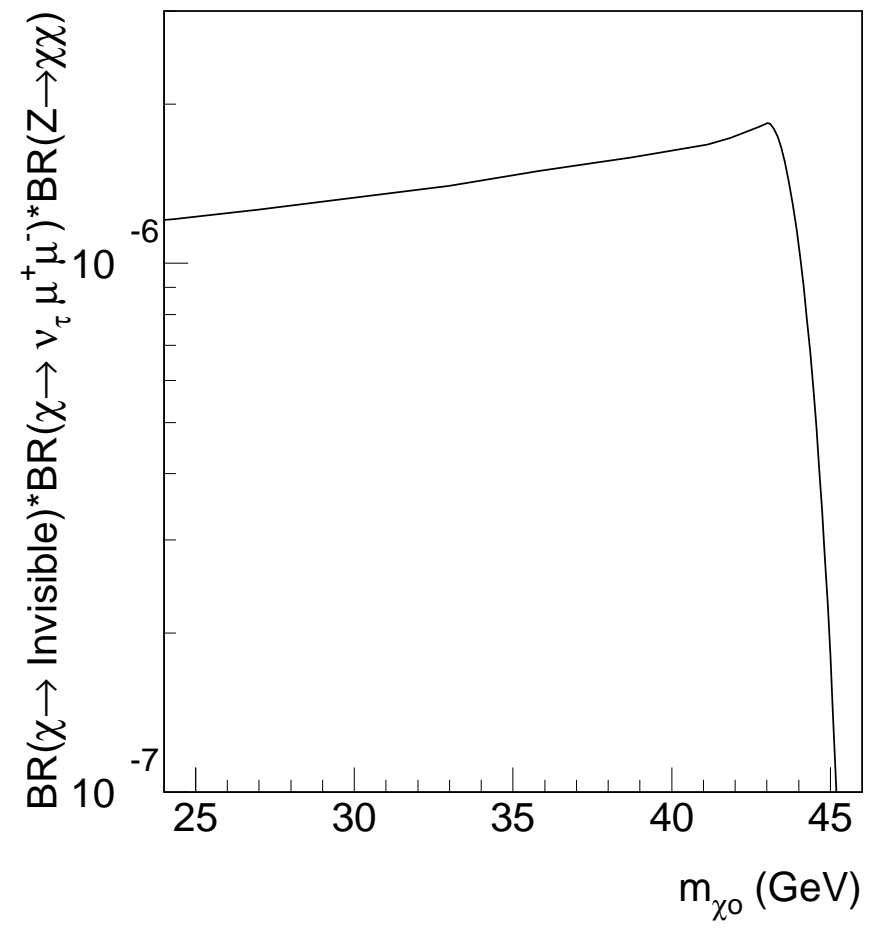

Figure 3: Region of sensitivity obtained at the $95 \%$ C.L. for $B R(Z \rightarrow \chi \chi)(\chi \rightarrow$ $\left.\mu^{+} \mu^{-} \nu\right) B R(\chi \rightarrow$ invisible $)$ as a function of the lightest neutralino mass. This is derived from searches of $p_{T}+\mu^{+} \mu^{-}$events that would arise from neutralino pair production at LEP, with one neutralino decaying invisibly and the other decaying as $\chi \rightarrow \mu^{+} \mu^{-} \nu$. 


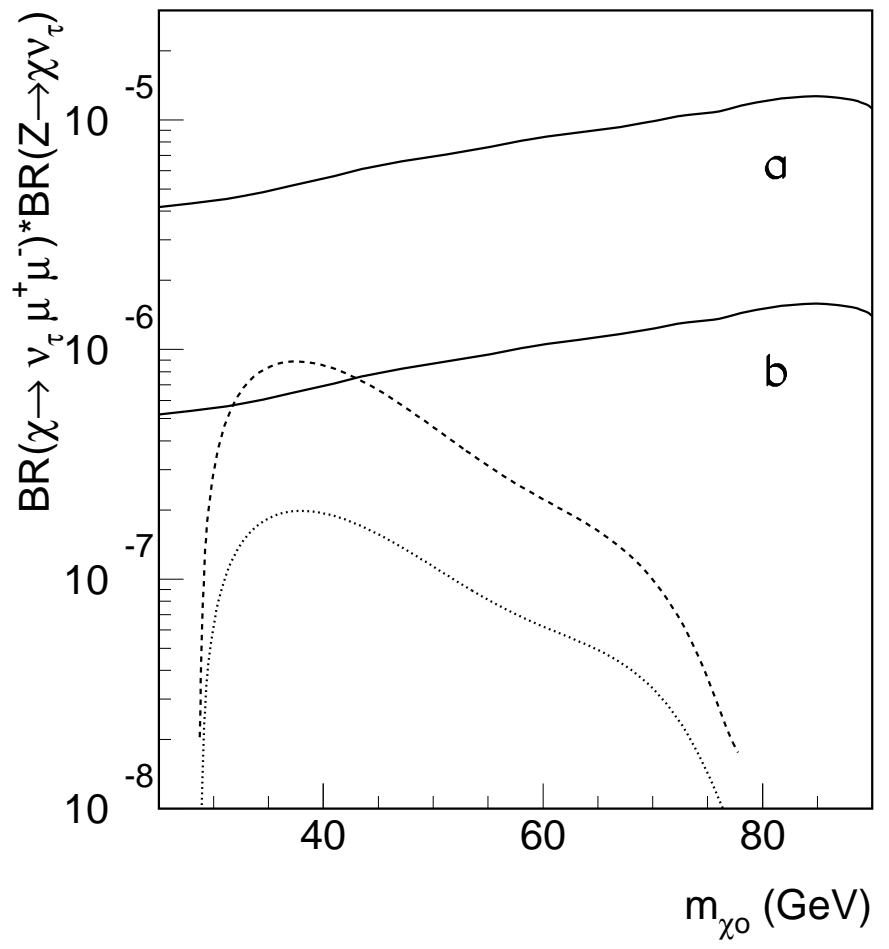

Figure 4: Comparison of the attainable limits on $B R(Z \rightarrow \chi \nu) B R\left(\chi \rightarrow \mu^{+} \mu^{-} \nu\right)$ versus the lightest neutralino mass, with the maximum theoretical values expected in different R-parity breaking models. The solid line (a) is the same as in Fig. 1, while (b) corresponds to the improvement expected from including the $e^{+} e^{-} \nu$ channel, as well as the combined statistics of the four LEP experiments. The dashed line corresponds to the model of ref. [16] allowing $m_{\nu_{\tau}}$ values as large as the present laboratory limit of ref. [23], while the dotted one is calculated in the spontaneous R-parity-violation model of ref. [5]. 


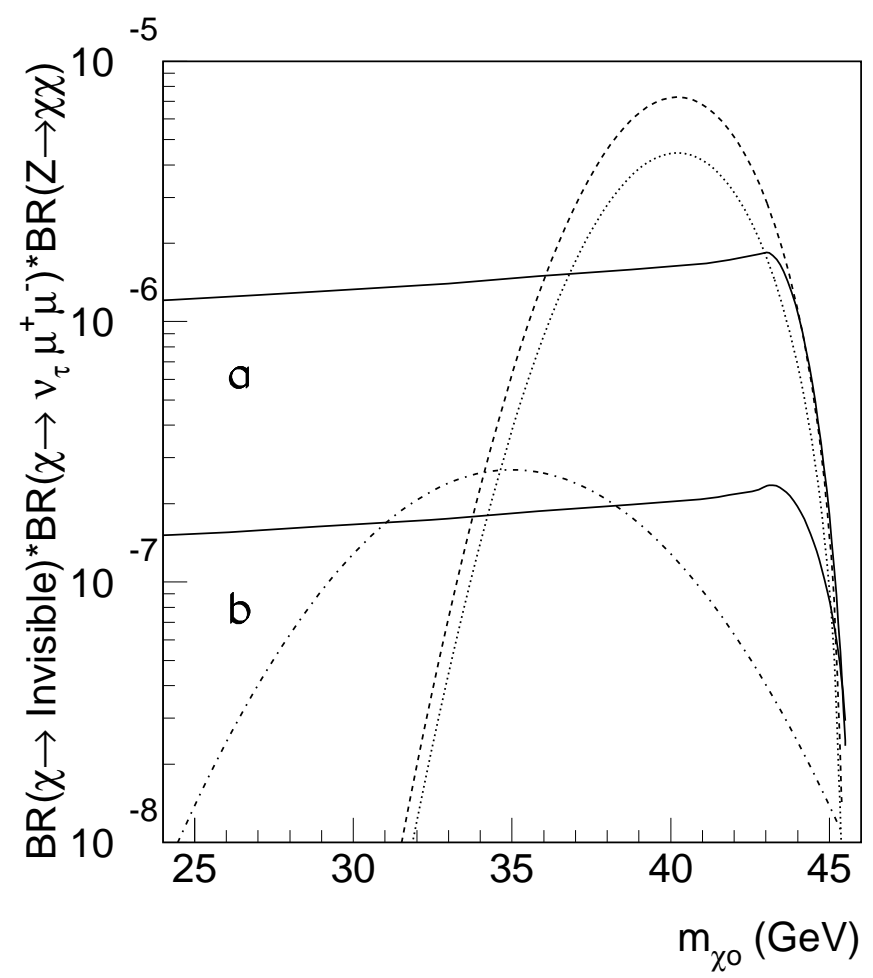

Figure 5: Comparison of the attainable limits on $B R(Z \rightarrow \chi \chi) B R\left(\chi \rightarrow \mu^{+} \mu^{-} \nu\right)$ versus the lightest neutralino mass, with the maximum theoretical values expected in different R-parity breaking models. The solid line (a) is the same as in Fig. 2, while (b) corresponds to the improvement expected from including the $e^{+} e^{-} \nu$ channel, as well as the combined statistics of the four LEP experiments. The dashed line corresponds to the model in ref. [16] allowing $m_{\nu_{\tau}}$ values as large as the present limit, the dotted one does implement the restriction on $m_{\nu_{\tau}}$ suggested by nucleosynthesis, and the dashdotted one is calculated in the model of ref. [5]. 


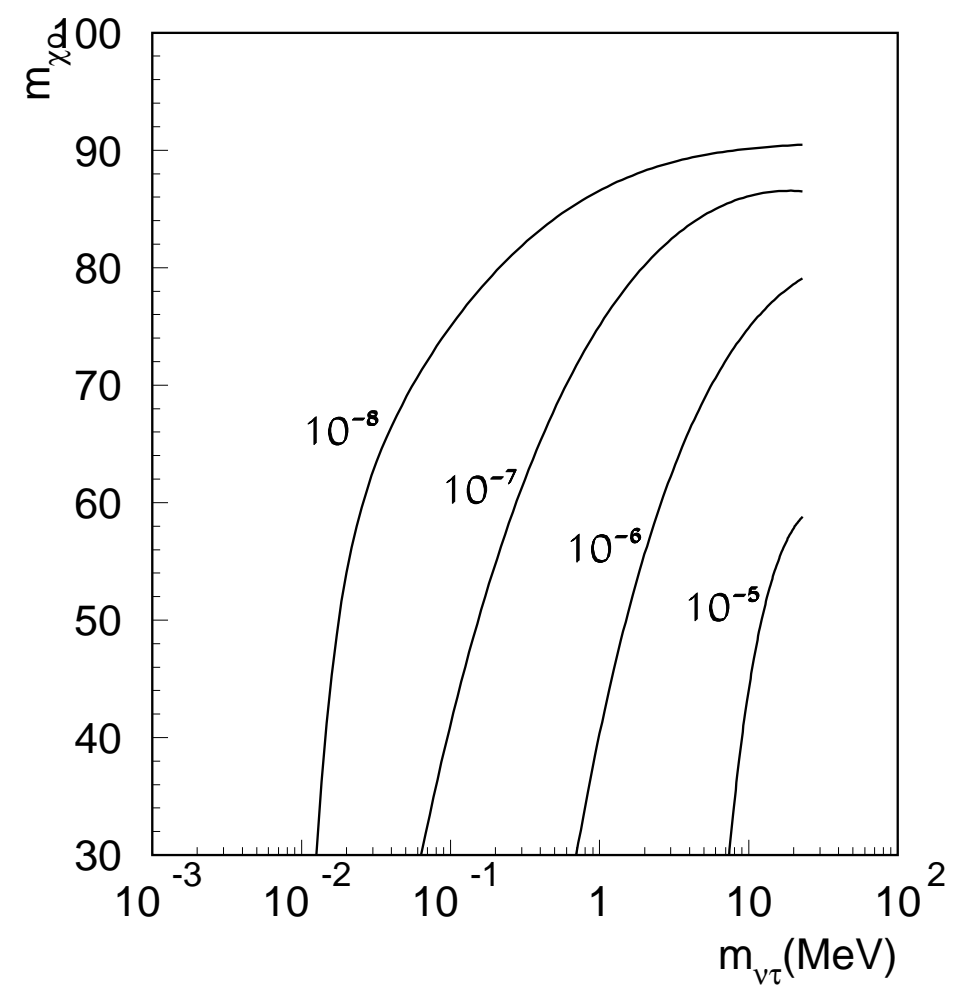

Figure 6: Attainable values for the R-parity breaking strength $B R(Z \rightarrow \chi \nu)$ versus the $\tau$-neutrino mass in the model of ref. [5]. 